\title{
EFFECTIVENESS OF DUAL ANTIBACTERIAL THERAPY AND EFFECTIVENESS OF RECOMMENDATION CRITERIA AS A DIAGNOSTIC TOOL IN CHILDREN WITH UNCOMPLICATED ACUTE APPENDICITIS
}

\author{
Liene Taurina*, Zane Liepina*, Astra Zviedre******, Arnis Engelis**,***, Aigars Petersons***,*** \\ * Faculty of Medicine, Rīga Stradinš University, Riga, Latvia \\ ** Department of Pediatric Surgery, Rīga Stradinš̌ University, Riga, Latvia \\ *** Department of Pediatric Surgery, Children's Clinical University Hospital, Riga, Latvia
}

\begin{abstract}
Summary
Introduction. Acute appendicitis (AA) - acute inflammation and infection of the appendix - is one of the most common cause for abdominal surgery in pediatrics. Appendectomy has been the gold standard since 1735, but in recent years there have been several studies that investigate antibacterial therapy for uncomplicated acute appendicitis. In 2016 new recommendations for suspected AA were implemented in emergency department setting of Children's Clinical University Hospital (CCUH).

Aim of the Study. To evaluate whether diagnostic criteria for patients with a suspected acute appendicitis is effective as a diagnostic tool and to evaluate whether dual antibacterial therapy is safe and effective as first line treatment for children with diagnosis of uncomplicated acute appendicitis in CCUH.
\end{abstract}

Material and methods. A retrospective study was made in CCUH from January 2017 to December 2017. Patients aged 7 to 18 with suspected uncomplicated acute appendicitis were identified and data were collected from an internal hospital management system and patients' medical records. Patients were divided into two groups - A and B. In group A were patients who met the criteria of uncomplicated AA, and in group $B$ - patients who met the criteria of complicated AA. Both groups received ampicillin and metronidazole. For statistical analysis IBM SPSS Statistics 22 program was used.

Results. From 98 patients majority were boys $(51 \%(n=50))$ with mean age 12.6 years. As a result of antibacterial therapy clinical condition improved in $93 \%(n=89)$ of patients who were discharged without surgery. 7 patients did not improve and underwent surgery within 48 hours after admission, another 2 had elective appendectomy. There was a significant positive moderate correlation between C-reactive protein (CRP), white blood cell count (WBC), basophil granulocytes (BASO) and appendix diameter at admisson and after 48 hours of dual antibacterial therapy ( $r=0.31, p=0.003$ vs. $r=0.37, p<0.001$ vs. $r=0.41, p=0.017$ vs. $r=0.51$, $\mathrm{p}=0.013)$. In group $\mathrm{A}(68 \%(\mathrm{n}=67))$ there was a significant positive moderate correlation between CRP, WBC and appendix diameter at admission and after 48 hours of dual antibacterial therapy ( $r=0.31, p=0.003$ vs. $r=0.37, p<0.001$ vs. $r=0.51, p=0.013)$. In group $B(30 \%(n=31)) 7$ patients underwent appendectomy and 2 patients received a broad spectrum antibacterial therapy prior to elective appendectomy. There is evidence of significant association between ALVARADO score $(p=0.004)$, rebound tenderness $(p<0.001), W B C(p=0.004), C R P(p<0.001)$ and stage in which appendicitis has progressed to.

Conclusions. Antibiotic-alone treatment may be a safe and effective initial management choice in children with uncomplicated acute appendicitis. However, to fully evaluate effectiveness of antibacterial therapy and diagnostic criteria, further follow-up research is needed.

A normal C-reactive protein value does not rule out uncomplicated acute appendicitis, therefore it can not act as a single marker to diagnose AA or to differentiate between complicated and uncomplicated AA.

Recommendations of treatment of UCAA are acceptable for use in emergency department as they act as a unified tool that allows pediatricians and pediatric surgeons to quickly assess suspected acute appendicitis.

Keywords:uncomplicated acute appendicitis, dual antibacterial therapy, nonsurgical management, pediatrics

\section{INTRODUCTION}

Acute appendicitis (AA) is acute inflammation and infection of the appendix. AA is one of the most frequent causes of abdominal pain and is the most common reason for abdominal surgery in children (14, 16). AA can be classified as uncomplicated (or simple) and complicated acute appendicitis by clinical severity at presentation. If appendectomy takes place, AA can then be divided further by pathological morphology catarrhal, phlegmonous, gangrenous and perforated appendicitis with or without abscess. Catarrhal and phlegmonous appendicitis falls under uncomplicated AA, but gangrenous and perforated appendicitis with or without abscess are classified as complicated AA (2, 
6). Appendicitis progresses through these stages and therefore delay in diagnosis and proper treatment of uncomplicated acute appendicitis (UCAA) can lead to perforation and emergent appendectomy.

Appendectomy has remained the treatment of choice for appendicitis for over a century $(15,11)$, but in recent years non-surgical management has proven to be a well established method $(5,13)$.

In 2017 there were 204 cases of AA, according to internal management system of Children's Clinical University Hospital (CCUH) and 98 of those were uncomplicated AA.

In recent years, opinions regarding the treatment of AA in CCUH has changed and new recommendations (see Figure 1) of suspected AA have been developed in emergency department setting (18). These recommendations are specifically designed for children aged 7 to 18 years old and they include ALVARADO score and values of Interleukin-6 (Il-6) and C-reactive protein (CRP). ALVARADO score was first developed in 1986 as a scoring system that determines probability of a patient having acute appendicitis (4). The score has 6 clinical items - rebound tenderness, migration of pain, anorexia, nausea and/or vomiting, right lower quadrant tenderness, fever $>37.3 \mathrm{C}$ and 2 laboratory measurements - leukocytosis $\left(>10 \times 10^{9}\right.$ L) and neutrophilic shift to the left $(>75 \%)$ with a total of 10 points (1). ALVARADO score, Il-6 and CRP are main and abdominal ultrasound (US) is additional criteria that enables division of patients with suspected AA into 2 groups - uncomplicated and complicated AA. It allows to determine further management.

\section{AIM OF THE STUDY}

To evaluate whether diagnostic criteria for patients with a suspected acute appendicitis is effective as a diagnostic tool and to evaluate whether dual antibacterial therapy is safe and effective as the first line treatment for children with diagnosis of uncomplicated acute appendicitis in CCUH.

\section{MATERIAL AND METHODS}

A retrospective study was made in CCUH from January 2017 to December 2017. Patients were identified by ICD-10 code K35 and data were collected from an internal hospital management system and patients' medical records.

This study is intended to evaluate symptoms and signs, laboratory and US findings among patients aged 7 18 years old admitted to Children's Clinical University Hospital emergency department with a diagnosis of uncomplicated acute appendicitis. All patients underwent dual antibacterial treatment with ampicillin and metronidazole.

Patients were divided into two groups - A and B. In group A were patients who met the criteria of uncomplicated acute appendicitis (ALVARADO score $\geq$ 7, negative rebound tenderness, CRP $\leq 8.4 \mathrm{mg} / \mathrm{L}$, white blood cell count $(\mathrm{WBC}) \leq 10.7 \times 10^{9} / \mathrm{l}$, appendix diameter in US $>7 \mathrm{~mm}$ ).
In group B were patients who met the criteria of complicated acute appendicitis (ALVARADO score $\geq 7$, positive rebound tenderness, CRP $>8.4 \mathrm{mg} / \mathrm{L}, \mathrm{WBC}>$ $10.7 \times 10^{9} / \mathrm{l}$, appendix diameter in US $>7 \mathrm{~mm}$ ).

Data were processed by using Microsoft Office Excel 2016 and IBM SPSS Statistics 22 programs (ShapiroWilk's Test, Fisher's Exact test, Pearson Chi - Square test, Spearman's Correlations Test, $\mathrm{p}<0.05$ ).

\section{RESULTS}

A total of 98 patients were included (boys $51 \%$ $(n=50))$. Mean age was 12.6 years $(S D=2.9) .93 \%$ of patients $(n=89)$ improved (patient demonstrates resolution of fever and pain, tolerance of general diet, occurance of spontaneous bowel movement and inflammatory marker values return to normal) with antibiotic treatment and were discharged within 3-10 days, without surgery (treatment success rate $89 \%) .7$ patients had no clinical improvement and had surgery within 48 hours after admission, another 2 underwent elective appendectomy after they were discharged: one after 3 weeks and the other after 9 weeks, respectively. No patient had allergic reactions to antibacterial treatment.

There was significant positive moderate correlation between CRP, WBC, basophil granulocytes (BASO) and appendix diameter at admisson and after 48 hours of dual antibacterial therapy $(\mathrm{r}=0.31, \mathrm{p}=0.003$ vs. $\mathrm{r}=0.37$, $\mathrm{p}<0.001$ vs. $\mathrm{r}=0.41, \mathrm{p}=0.017$ vs. $\mathrm{r}=0.5 \mathrm{l}, \mathrm{p}=0.013$ ) (see Figures $2-5$ ). There was not a significant correlation between neutrophils (NEUT) before and after antibacterial treatment $(\mathrm{r}=-0.004, \mathrm{p}=0.979)$. There was a significant positive moderate correlation between duration of symptoms and CRP at admission ( $r=0.380$, $\mathrm{p}<0.001)$.

In group $A$, there were $68 \%(n=67)$ patients who met the criteria of UCAA; a significant positive moderate correlation was observed between CRP, WBC and appendix diameter at admission and after 48 hours of dual antibacterial therapy ( $\mathrm{r}=0.31, \mathrm{p}=0.003 \mathrm{vs}$. $r=0.37, p<0.001$ vs. $r=0.51, p=0.013)$. In group $B(30 \%$ $(\mathrm{n}=31)) .7$ patients underwent appendectomy and 2 patients received broad spectrum antibacterial therapy prior to elective appendectomy.

There is evidence of significant association between ALVARADO score $(\mathrm{p}=0,004)$, rebound tenderness $(\mathrm{p}<0.001)$, WBC $(\mathrm{p}=0.004), \mathrm{CRP}(\mathrm{p}<0.001)$ and type of diagnosis.

\section{DISCUSSION}

Appendectomy has been standard treatment in AA since 1735 (11). However in recent years, conservative therapy of acute appendicitis with antibiotics has become more popular. The reason why paradigm shift occurs is because conservative treatment compared to surgical treatment allows to avoid from both surgical and general anesthesia as well as related complications of either. One more important aspect of non-operative management is low recurrence rate - previously done studies indicate $14 \%$ recurrence (5) and a report done 
three years earlier in CCUH shows $15 \%$ recurrence rate (12). Also, there is a notion that the appendix may act as a reservoir for normal gut flora, enabling the large bowel microbiome to recover more rapidly after severe gastroenteritis (10).

CCUH is the only hospital in Latvia where children with UCAA are cured by pediatric surgeons following the aforementioned recommendations. The reasons for it are growing antibiotic resistance (8) and in case of failure of antibacterial treatment patient has direct access to advanced medical approach.

In CCUH standard antibacterial regimen is empiric and includes ampicillin and metronidazole, but in several studies done previously pediatric surgeons use broadspectrum antibiotics, such as piperacillin-tazobactam $(9,7)$ or meropenem and metronidazole $(15)$. Cameron et al. completed a study where effectiveness of broad versus narrow-spectrum antibiotics were compared and it was concluded that narrow-spectrum antibiotics were just as effective as broad-spectrum antibiotics (3). According to the results of this present study it can be concluded that narrow-spectrum antibiotics have $89 \%$ treatment success rate. It could be attributed to the fact that these results are obtained during inpatient therapy, therefore further research with follow-up is necessary.

There are certain possible aspects that could affect the results. The lack of standardized protocol for description of US findings was one of the main limitations. It is also critical to note that US is operator dependent investigation. Furthermore, detection of serum Il-6 is a paid service thereby there were insufficient data in medical records about Il-6 and it was not analyzed in this study.

Despite the fact that WBC and CRP are nonspecific inflammatory markers with variable sensitivity and specificity (17), they can be used as indicators to control dynamics of therapy and to determine clinical improvement. Nevertheless, it is important to remember that AA is occasionally present despite a normal WBC and CRP (14).

\section{CONCLUSIONS}

In the case of acute uncomplicated appendicitis, the option of antibiotic-only treatment may be a safe and effective initial management choice for children as their laboratory, ultrasound findings, and overall clinical status improve. However, to fully evaluate the effectiveness of antibacterial therapy and diagnostic criteria further follow-up research is needed.

Normal C-reactive protein values do not rule out uncomplicated acute appendicitis. Therefore it can not act as a single marker to diagnose AA or to differentiate between complicated and uncomplicated AA. It would be valuable to continue to search for more specific, sensitive and cost-effective markers.

Recommendations of treatment of UCAA are acceptable for use in emergency department as they act as a unified tool that allows pediatricians and pediatric surgeons to quickly assess suspected acute appendicitis. Also, validation of the use of these recommendations after further prospective studies could be considered for a wide range of physicians such as general practitioners and colleagues in Regional Hospitals.

Conflict of interest: None 


\section{Recommendations for patients with suspected acute appendicitis}

\section{$<7$ years old}

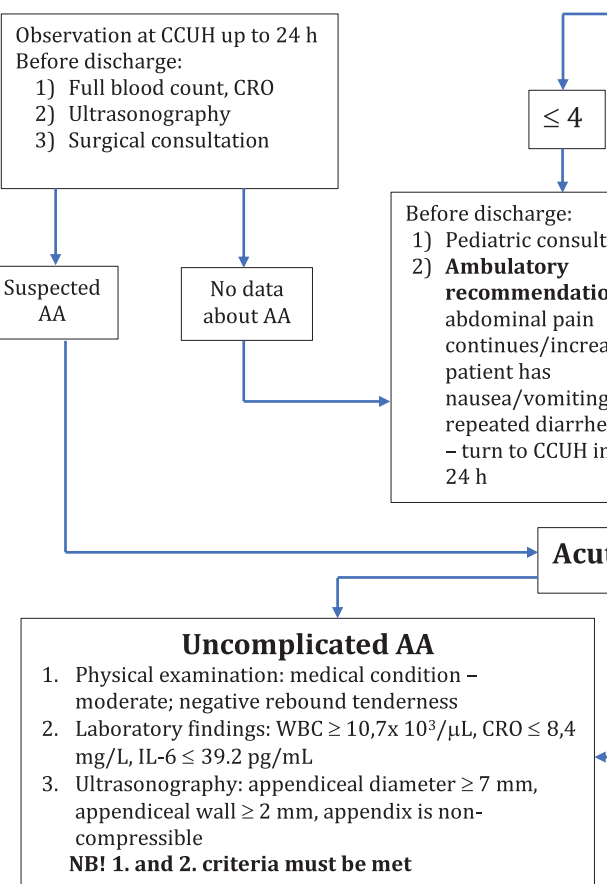

$\geq 7-18$ years old

\section{ALVARADO score + IL-6 (only for first $24 \mathrm{~h}$ ),}

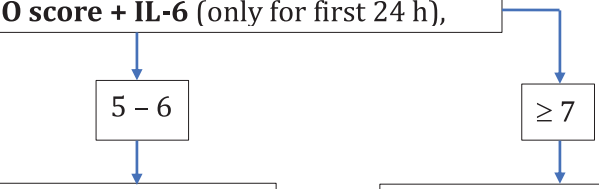

Observe at CCUH for $24 \mathrm{~h}$ Before discharge:

1) Full blood count, CRP

2) Ultrasonography

3) Surgical consultation

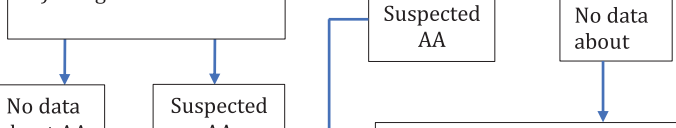

Observe at CCUH for $24 \mathrm{~h}$ Before discharge:

1) Surgical consultation

2) Full blood count, CRP

3) Ultrasonography

4) Pediatric consultation

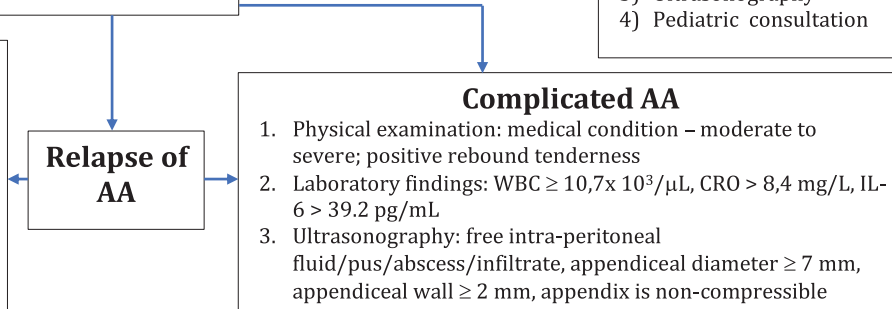

Fig. 1. Algorithm for patients with suspected acute appendicitis in Children's Clinical University Hospital

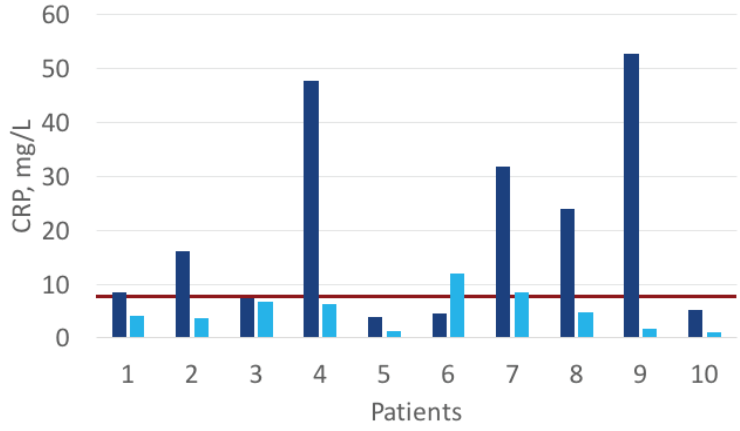

CRP before treatment $\quad$ CRP after treatment

Fig. 2. Tendency of serum CRP values before and after antibacterial treatment $(p=0.003$, Spearman's Correlations Test)

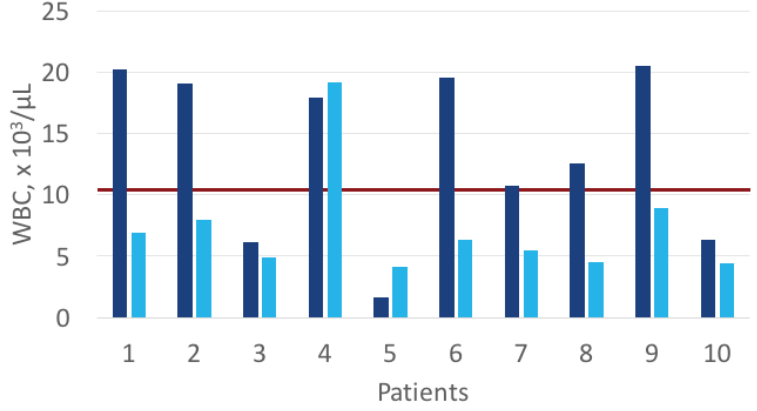

- WBC before treatment WBC after treatment

Fig. 3. Tendency of serum WBC values before and after antibacterial treatment $(p<0.001$, Spearman's Correlations Test) 


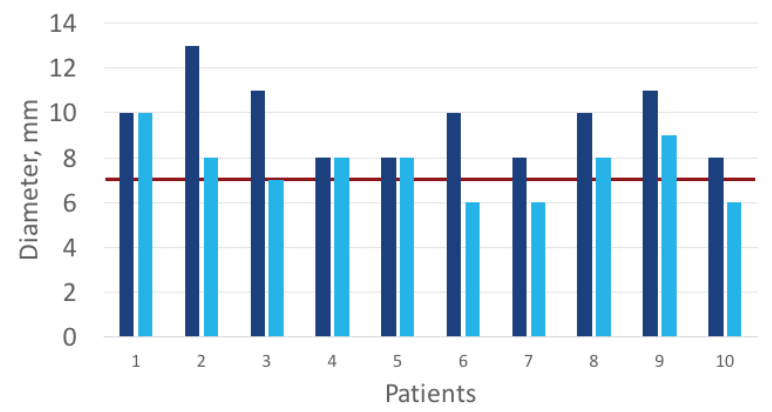

- Diameter before treatment Diameter after treatment

Fig. 4. Tendency of diameter of appendix in US before and after antibacterial treatment $(p=0.013$, Spearman's Correlations Test)

\section{REFERENCES}

1. Alvarado A. A practical score for the early diagnosis of acute appendicitis // Ann Emerg Med, 1986; 15 (5): 557-564

2. Bhangu A, Søreide K, Di Saverio S, Assarsson JH, Drake FT. Acute appendicitis: modern understanding of pathogenesis, diagnosis, and management // Lancet, 2015; 386:1278-87

3. Cameron DB, Melvin P, Graham DA, Glass CC, Serres SK, Kronman MP, Saito JM, Rangel SJ. Extended Versus Narrow-spectrum Antibiotics in the Management of Uncomplicated Appendicitis in Children: A Propensity-matched Comparative Effectiveness Study // Ann Surg, 2018; 268(1): $186-192$

4. Fasen G, Schirmer B, Hedrick TL. Appendix // Yeo CJ. Shackelford's Surgery of the Alimentary Tract, Vol.2. 8th ed. Philadelphia: Elsevier; 2019; 1951 1958

5. Georgiou R, Eaton S, Stanton MP, Pierro A, Hall NJ. Efficacy and safety of nonoperative treatment for acute appendicitis: A meta-analysis // Pediatrics 2017; 139(3):e20163003

6. Haijanen J, Sippola S, Grönroos J et al. Optimising the antibiotic treatment of uncomplicated acute appendicitis: a protocol for a multicentre randomised clinical trial (APPAC II trial) // BMC Surg, 2018; 18:doi:10.1186/s12893-018-0451-y

7. Hartwich J, Luks FI, Watson-Smith D, et al. Nonoperative treatment of acute appendicitis in children: a feasibility study // J Pediatr Surg, 2016; 51(1):111- 116

8. Mak GZ, Leoff DS. Paradigm shifts in the treatment of appendicitis // Pediatr Ann, 2016; 45:235 - 240

9. Minneci PC, Mahida JB, Lodwick DL, et al. Effectiveness of patient choice in nonoperative vs surgical management of pediatric uncomplicated acute appendicitis // JAMA Surg, 2016; 151(5): $408-415$

10. Randal BR, Barbas AS, Bush EL, Lin SS, Parker W. Biofilms in the large bowel suggest an apparent function of the human vermiform appendix // J Theor Biol, 2007; 249:826 - 831

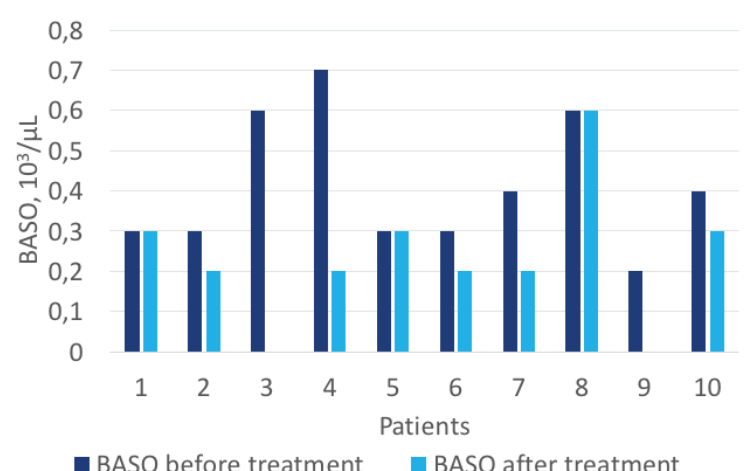

Fig. 5. Tendency of serum basophil values before and after antibacterial treatment $(p=0.017$, Spearman's Correlations Test)

11. Richmond B. The Appendix // Townsend CM, Beauchamp RD, Evers BM, Mattox KL. Sabiston Textbook of Surgery. 20th ed. Philadelphia: Elsevier; 2017; $1296-1311$

12. Silina E, Jurgele A, Viksne A, Abola Z, Engelis A, Petersons A. Nonsurgical Management of Acute Uncomplicated Appendicitis in Children: The Analysis of Treatment Outcome in Relationship with Antimicrobial Regimens and Adverse Prognostic Factors // Acta Chir Latviensis, 2016; $16: 12-16$

13. Steiner Z, Buklan G, Gutermacher M, Litmanovitz I, Landa T, Arnon S. Conservative antibiotic treatment for acute uncomplicated appendicitis is feasible // Pediatr Surg Int, 2018; 34(3):283 - 288

14. Stringer MD. Acute appendicitis // J Paediatr Child Health, 2017; 53:1071 - 1076

15. Svensson JF, Patkova B, Almstrom M, et al. Nonoperative treatment with antibiotics versus surgery for acute nonperforated appendicitis in children: a pilot randomized controlled trial // Ann Surg, 2015; 261(1):67-71

16. Yamanaka S, Skarsgard ED, Goldman RD. Conservative therapy for appendicitis in children // Can Fam Physician 2018; 64(8):574 - 576

17. Yu CW, Juan LI, Wu MH, Shen CJ, WU JY, Lee CC. Systematic review and meta-analysis of the diagnostic accuracy of procalcitonin, C-reactive protein and white blood cell count for suspected acute appendicitis // Br J Surg, 2013; 100(3): $322-329$

18. Zviedre A. Correlation of Acute Appendicitis and Acute Mesenteric Lymphadenitis With the Changes of Serum Cytokines in Children // Summary of Doctoral Thesis, 2016; Riga; Rīga Stradiņš University

\section{Address:}

Liene Taurina

Rīga Stradiņš University

Dzirciema Street 16, Riga, LV-1007

liene.stura@gmail.com 